\title{
The role of the complement cascade in endotoxin-induced septic encephalopathy
}

\author{
Alexander Jacob, Lauren K Hensley, Bryan D Safratowich, Richard J Quigg and Jessy J Alexander
}

The complement system normally eliminates bacteria and has a protective effect. However, in an inflammatory setting such as sepsis, an exaggerated or insufficient activation of this cascade can have deleterious effect through the activation of glial cells, secretion of proinflammatory cytokines and generation of other toxic products. The aim of the present study was to investigate the role of the complement cascade in septic encephalopathy, through the passive injection of endotoxin/lipopolysaccharide (LPS) into mice overexpressing the potent complement inhibitor, CR1-related y (Crry-tg). Increased gliosis occurred in brains of endotoxemic mice. Concomitant with this, there was a significant rise in mRNA expression of GFAP, CD45 and proinflammatory molecules, TLR4, TNF- $\alpha$ and NO, in these brains. Consistent with the capacity of these inflammatory mediators, there was increased apoptosis as determined by DNA fragmentation and TUNEL staining on LPS treatment, which occurred through the Akt pathway. In addition, there was increased water content in brain, similar to cerebral edema observed in sepsis. Relative to wild-type mice, complement-inhibited mice had an attenuated inflammatory response, decreased edema and reduced apoptosis. Therefore, we demonstrate for the first time that the complement cascade appears to be one of the key players that cause brain pathology in an endotoxemic setting and therefore is a viable therapeutic target.

Laboratory Investigation (2007) 87, 1186-1194; doi:10.1038/labinvest.3700686; published online 8 October 2007

KEYWORDS: apoptosis; complement; lipopolysaccharide; neuroimmunology; sepsis

Septic encephalopathy is the consequence of the body's response to infection. It may be due to multiple factors including inflammatory cells and their mediators, reduced cerebral blood flow, disruption of the blood-brain barrier (BBB), cerebral edema and inflammation. ${ }^{1,2}$ Although the exact mechanism of injury remains undefined, it is clear that inflammatory processes play a significant role and are important therapeutic targets. Clinical and experimental studies indicate that complement activation could contribute to inflammation, leukocyte recruitment, neuronal cell death and BBB dysfunction. ${ }^{3-5}$ Although complement activation has been documented in septic shock models ${ }^{6-8}$ and in septic humans, ${ }^{9-11}$ its role in central nervous system endotoxemia still remains unclear and is the focus of this study.

Complement activation leads to cleavage of the key proteins, C3 and C5. C3b generated binds to immune complexes and C5b initiates the assembly of the C5b-9 complex (MAC) that can result in cellular death or activation. ${ }^{12}$ To prevent self injury, regulatory proteins strictly control the spontaneous and immune complex-induced activation of the complement system. CR1-related y (Crry) ${ }^{13,14}$ is a functional homologue of decay-accelerating factor and membrane cofactor protein and regulates both the classical and alternative complement pathways in mice. ${ }^{15}$ Complement activation can also alter the release of cytokines and cause cell necrosis or apoptosis in endotoxemic brains. The complement system along with other inflammatory cytokines can lead to edema. ${ }^{1}$ Water content in the brain is tightly regulated by the water channel protein aquaporin $4(\mathrm{AQP})$ present in astrocytic end feet ${ }^{16,17}$ and reactive microglia. ${ }^{18}$ In addition, neutrophil infiltration overlaps with edema development in experimental stroke, and has been implicated in the generation of vasogenic cerebral edema. ${ }^{19-23}$

Complement proteins are synthesized by the different cellular fractions in the brain, including the microglia and astrocytes. Microglia and astrocytes are the resident immunoeffector cells of the central nervous system. These cells are quiescent under normal conditions. Upon activation their

Department of Medicine, University of Chicago, Chicago, IL, USA

Correspondence: Dr JJ Alexander, PhD, Department of Medicine, University of Chicago, 5841 South Maryland Avenue, MC 5100, Chicago, IL 60637, USA.

E-mail: jalexand@medicine.bsd.uchicago.edu

Received 24 April 2007; revised 6 September 2007; accepted 6 September 2007 
morphology, immunophenotype and expression pattern of inflammatory mediators changes, leading to immune and inflammatory responses.

Lipopolysaccharide (LPS), the cell-wall component of Gram-negative bacteria, induces sepsis with a number of similar pathological sequelae in humans and animals. ${ }^{24-27}$ Therefore, we used the LPS model to determine the role of the complement causing apoptosis and edema in brain during sepsis. We assessed the proinflammatory cytokines, TNF- $\alpha$ and TLR4 expression, and the expression of effector enzymes such as inducible nitric oxide synthase (iNOS). In addition, using immunohistochemical staining and quantification of terminal deoxynucleotidyl transferase-mediated biotinylated UTP nick end labeling (TUNEL)-positive cell death after intraperitoneal LPS challenge. In our studies, LPS treatment induced complement activation, leading to upregulation of CD45, TNF- $\alpha$ and TLR4. We also saw an increase in apoptosis and edema, suggesting that complement may be a viable therapeutic target for sepsis.

\section{MATERIALS AND METHODS Animals and Treatments}

C57BL/6 mice that overexpress Crry directed by the metallothionein-I promoter were generated in the laboratory ${ }^{13,28}$ In all the animals, the presence of the Crry transgene was documented by polymerase chain reaction (PCR), and soluble Crry in sera was identified by enzyme-linked immunosorbent assay (ELISA). Mice were used for experiments at 8 weeks of age. The animals were maintained on $12 \mathrm{~h} \mathrm{light}$ and dark cycles with free access to food and water. One group of Crry-tg mice were given LPS (from Escherichia coli serotype 055:B55; Sigma Aldrich, St Louis, MO, USA, Lot no.127H4097) as a single injection of $0.5 \mathrm{mg}$ i.p., and another group received the saline vehicle as control. The mice were killed $8 \mathrm{~h}$ later and their brains were harvested. All experiments were performed in accordance with the guidelines set by the University of Chicago Institutional Animal Care and Use Committee.

\section{Tissue Processing}

Brains were isolated from animals immediately upon killing. Cerebellum and brain stem were discarded. The anterior region of the cerebral cortex was snap frozen for immunofluorescence (IF) microscopy and the remainder was processed for RNA and genomic DNA isolation.

\section{Brain Water Content}

The brains were removed, weighed immediately and then kept at $300^{\circ} \mathrm{C}$ for $48 \mathrm{~h}$. The brains were weighed during this process at $24 \mathrm{~h}$ and then again at $48 \mathrm{~h}$ to make sure they had reached a consistent weight. The percentage of brain water in the tissue was calculated as (wet weight-dry weight) $\times 100 /$ wet weight.

\section{TUNEL Staining}

TUNEL staining was performed with the TdT-FragEL DNA fragmentation detection kit (Oncogene) according to the manufacturer's instructions. In brief, $8-\mu \mathrm{m}$ cryostat sections were washed in Tween 20/TBS and fixed in 4\% formaldehyde for $30 \mathrm{~min}$. This was followed by proteinase $\mathrm{K}$ treatment for $10 \mathrm{~min}$ and incubation in $3 \% \mathrm{H}_{2} \mathrm{O}_{2} /$ methanol for $7 \mathrm{~min}$. Specimens were incubated with biotin-TdT at $37^{\circ} \mathrm{C}$ for $60 \mathrm{~min}$, blocked in dilute BSA for $20 \mathrm{~min}$ and incubated in streptavidin-horseradish peroxidase for $30 \mathrm{~min}$, followed by detection with diaminobenzidine reagent for $15 \mathrm{~min}$. Sections were counterstained with methyl green and a blinded observer counted the number of positively stained nuclei per high-power field and recorded the average of 10 fields for each sample. For dual localization studies by IF, a similar protocol was followed using the TACS 2 TdT-Fluor reagent kit (Trevigen). In this instance, biotin-TdT in apoptotic nuclei was identified with streptavidin-FITC and neurons were stained with rabbit anti-mouse neurofilament $\mathrm{Ab}$ (Chemicon International), followed by rhodamine-conjugated anti-rabbit IgG (Sigma Aldrich).

\section{Ligase-Mediated-PCR}

Brains were harvested and immediately frozen at $-80^{\circ} \mathrm{C}$. DNA was purified using the DNeasy DNA purification system (Qiagen, Valencia, CA, USA). DNA laddering was detected by ligase-mediated-PCR (LM-PCR) (Clontech Laboratories, Palo Alto, CA, USA) according to the manufacturer's instructions. In brief, DNA isolated from each animal was incubated with the supplied primer targets and T4 DNA ligase for $18 \mathrm{~h}$ at $16^{\circ} \mathrm{C}$. A $20-\mu \mathrm{g}$ weight of this ligated DNA was then used as substrate for PCR, using the supplied primers and Advantage DNA polymerase (Clontech Laboratories) for 23 cycles at $94^{\circ} \mathrm{C}$ for $1 \mathrm{~min}$ and at $72^{\circ} \mathrm{C}$ for $3 \mathrm{~min}$. The reaction product for each animal was electrophoresed through a $1.2 \%$ agarose gel with ethidium bromide and visualized with UV light.

\section{Tissue Staining}

Cryostat sections $(4 \mu \mathrm{m})$ were fixed with ethanol:ether and ethanol and direct immunofluorescence microscopy was performed using FITC-conjugated antibodies to mouse C3 (Cappel Laboratories, Durham, NC, USA), glial fibrillary acidic protein (GFAP; Dako, High Wycombe, UK), neuronspecific nuclear protein (NeuN) (Chemicon) and tomato lectin (Sigma). Sections were examined using a BX-60 IF microscope (Olympus Optical Co., Japan) equipped with epifluorescence optics for the detection of fluorescein and rhodamine ( $\times 20$ magnification).

\section{Quantitative RT-PCR}

RNA from brains was isolated using TriZol reagent (Life Technologies, Grand Island, NY, USA). Samples were then treated with RNAse-free DNAse (Promega, Madison, WI, USA) and the DNAse inactivated with EGTA as per the 
manufacturer's instructions. Real-time quantitative (q)RTPCR was performed for CD45, TNF- $\alpha$ and TLR4. cDNA was generated from RNA using random hexamers with the SuperScript first-strand synthesis kit (Life Technologies). qPCR was performed using a Smart Cycler (Cepheid, Sunnyvale, CA, USA). Each reaction was conducted in a total volume of $25 \mu \mathrm{l}$ using the SYBRGreen intercalating dye method (PE Applied Biosystems), ${ }^{3}$ cDNA, primers at $200 \mathrm{nM}$ each, and probe at $100 \mathrm{nM}$. PCR was conducted at an annealing temperature of $60^{\circ} \mathrm{C}$. For each sample, the number of cycles required to generate a given threshold signal $\left(C_{\mathrm{t}}\right)$ was recorded. Standard curves were generated for the gene of interest from serial dilutions of splenic cDNA, and the ratio of gene expression relative to GAPDH expression was calculated for each experimental animal. Measurements of individual mRNA expression relative to GAPDH were performed in a similar manner. Primers were synthesized by Integrated DNA Technologies (Coralville, IA, USA) and probes by Synthegen (Houston, TX, USA). The sequences of primers/probes are shown in Table 1.

\section{Western Blotting}

For immunoblotting, equivalent amounts of proteins, quantified by BCA assay, were separated on 10\% SDS-polyacrylamide gels ( $30 \mu \mathrm{g}$ per lane) and probed with rabbit anti-mouse C3 at 1:1000 (Sigma), rabbit anti-mouse phosphor-Akt (Ser 473), Akt, or TLR4 as per the manufacturer's instructions (Cell Signalling Technology, MA, USA). To control for equal sample loading, we also performed immunoblotting with an antibody against the housekeeping gene, actin, at 1:1500 (Sigma). Horseradish peroxidaselabeled secondary antibodies (rabbit anti-goat, 1:5000 (Sigma) or goat anti-rabbit, 1:1500 (Pierce)) were used. Bound antibody was detected by the enhanced chemiluminescence method (Amersham Biosciences, Piscataway, NJ, USA). To remove bound antibodies, the immunoblots were treated with stripping buffer (Bio-Rad) as per the manufacturer's instructions. The efficacy of the stripping procedure was confirmed by detecting the stripped blot with secondary antibody alone to ensure that no bound antibodies could be detected.

\section{Statistics}

All data are expressed as means \pm s.d. and were analyzed using Minitab software (State College, PA, USA). When a single experimental group was compared with its littermate

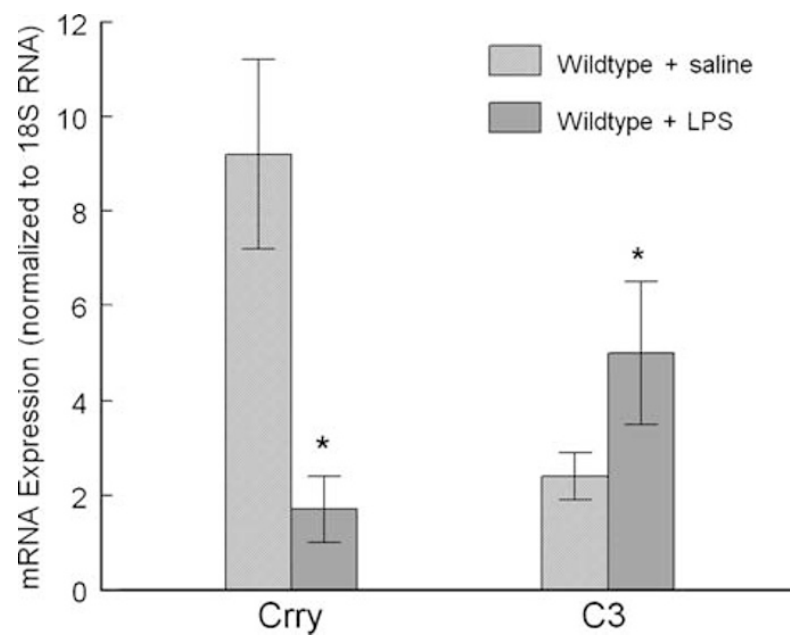

Figure 1 Increased mRNA expression of $\mathrm{C} 3$ and reduced expression of Crry levels in endotoxemia. mRNA expression of $\mathrm{C} 3$ and Crry was determined by qRT-PCR and normalized to the expression of $18 \mathrm{~S}$. At killing, $8 \mathrm{~h}$ after LPS treatment, there was a profound increase in C3 expression and a significant decrease in Crry expression in the brain. Values are given as means \pm s.d. ( $n=6$ per group); ${ }^{\star} P<0.01$ vs saline treated.

Table 1 The sequences of primers and probes used in qRT-PCR were:

\begin{tabular}{llll}
\hline Gene & $\begin{array}{l}\text { Forward primer } \\
5^{\prime}-\cdots-3^{\prime}\end{array}$ & $\begin{array}{l}\text { Reverse primer } \\
5^{\prime}-\cdots-3^{\prime}\end{array}$ & $\begin{array}{l}\text { Probe } \\
5^{\prime}-\cdots-3^{\prime}\end{array}$ \\
\hline $\begin{array}{l}\text { FAM/TAMRA dyes } \\
\text { GAPDH }\end{array}$ & & \\
INOS & GGCAAATCAAGGCACAGT & AGATGGTGATGGGCTTCCC & AGGCCGAGAATGGGAAGCTTGTCATC \\
& CAGCTGGGCTGTACAACCTT & TGAATGTGATGTTGCTTCGG & CGGGCAGCCTGTGAGACCTTGA
\end{tabular}

SYBRGreen incorporation

$\mathrm{C} 3$

CD45

TNF- $\alpha$

TLR4

AQP4

185
AGAGGCAAGTGCTGACCAGT

CAGAGCATTCCACGGGTATT

CCGATGGGTTGTACCTTGTC

ACCTGGCTGGTTACACGTC

AGGTGCCCTITATGAGTATG

GTAACCCGTTGAACCCCATT
GGCAGCACGTATTCGTTCAC

GGACCCTGCATCTCCATTTA

GTGGGTGAGGAGCACGTAGT

CAGGCTGTITGTTCCCAAAT

СТTCССTСТTСТСТTСТCC

CCATCCAATCGGTAGTAGCG

AQP4, aquaporin 4; C3, complement 3; FAM, 6-carboxy-fluorescein; GAPDH, glyceraldehyde-3-phosphate dehydrogenase; iNOS, inducible nitric oxide synthase; TAMRA, 6-carboxy-teremethyl-rhodamine; TLR4, toll-like receptor 4; TNF- $\alpha$, tumor necrosis factor $\alpha$. 

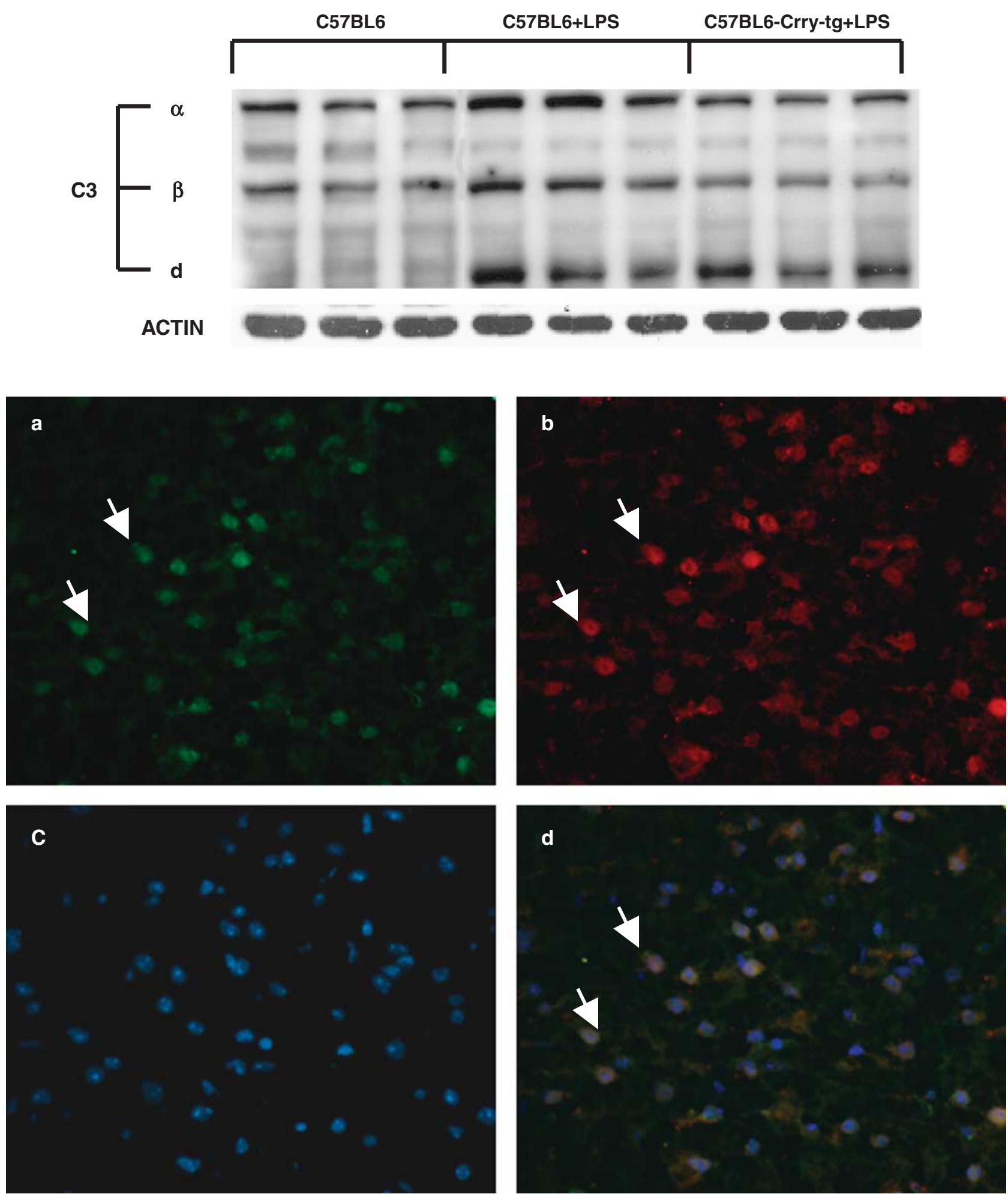

Figure 2 LPS induces increased C3 expression in the brain. The amount of C3 was assessed in brain by western blotting and normalized to actin. Representative samples (three from each group, $n=6$ ) are shown here. C3 was significantly increased in LPS-treated mice compared with controls. This increase was prevented by complement inhibition. Representative sections from C57BL6 mice treated with LPS were stained for C3 (a) and NeuN (b). (c) Nuclei were stained using DAPI. The merged picture (d) indicates that C3 deposits were mainly found in the neurons. 
control, two sample $t$-tests were used. For multiple comparisons, one-way ANOVA followed by Tukey's pairwise comparisons was used.

\section{RESULTS}

\section{Expression of Complement Proteins, C3 and Crry in Brain is Altered in Endotoxemia}

Crry is a potent regulator of the complement cascade, affecting both the classical and alternative pathways. The mRNA expression of Crry was reduced after LPS injection into wild-type mice. In contrast, the expression of the key protein C3 was increased in LPS-treated mice compared with controls (Figure 1).

\section{C3 Expression is Significantly Increased in LPS-Treated Brains}

We examined the effect of complement inhibition in LPStreated mice, which produced sCrry endogenously by a constitutively active transgene. Expression of the key complement protein C3 was significantly increased in LPS-treated mouse brains (Figure 2). As expected in the setting of complement inhibition with Crry, there was less C3 in the brains of Crry transgenic mice compared with transgene-negative controls. Samples were processed on a reducing gel. Complement activation results in the production of $\mathrm{C} 3$ breakdown products (Figure 2). Merged immunofluorescence image (Figure 2d) indicates the localization of C3 deposits (Figure 2a) in the cortical neurons (Figure 2b).

\section{Endotoxemia Leads to Upregulated Inflammatory Mediator Expression in Brain}

Since inflammation is an important feature of sepsis, we used qRT-PCR to quantify the expression of mRNA for key inflammatory mediators, CD45, TNF- $\alpha$ and TLR4 in the brains of LPS-treated mice, and also to see how this was affected by complement inhibition. In mice treated with LPS, there was a twofold increase in CD45 and TNF- $\alpha$ mRNA, and a threefold increase in TLR4 mRNA, all of which were statistically different from the control mice given the vehicle alone (Figure 3). Complement inhibition prevented the LPS-induced increase in the expression of these inflammatory mediators.

\section{LPS Upregulates iNOS in a Complement-Dependent Manner}

$\mathrm{NO}$ is an integral mediator of a wide range of inflammatory as well as anti-inflammatory processes. The isoform responsible for NO production during inflammation is the calcium-independent iNOS. ${ }^{29}$ iNOS has been shown to be strongly upregulated in a wide variety of tissues, including the brain, after the administration of LPS. ${ }^{30}$ Further, increased iNOS activity was found in circulating neutrophils of septic shock patients compared with healthy volunteers. ${ }^{31}$ Since infiltration of neutrophils was significantly increased in LPS-treated mice, we assessed the expression of iNOS mRNA in the brain. LPS markedly upregulated iNOS expression in

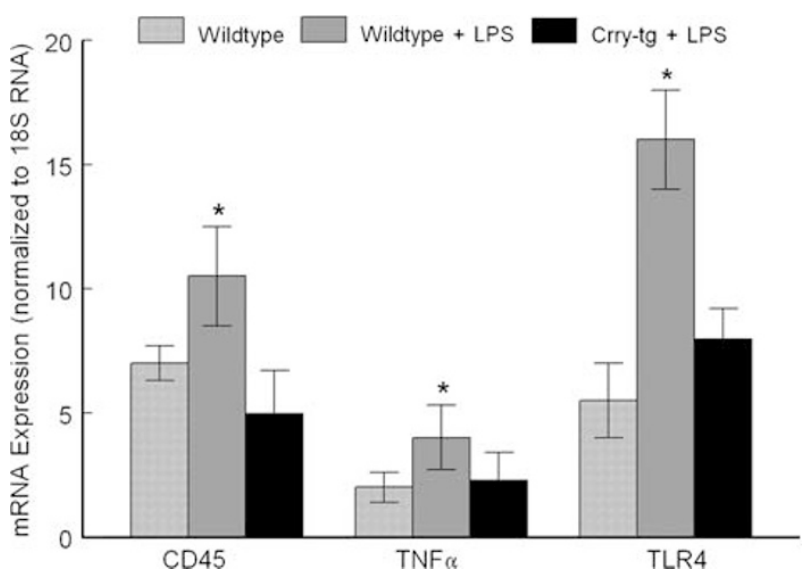

Figure 3 Brain expression of inflammatory mediators is upregulated in endotoxemia. Expression of CD45, TLR4 and TNF- $\alpha$ was significantly upregulated in the brains of endotoxemic mice, as determined by qRT-PCR, normalized to $18 \mathrm{~S}$ expression. These alterations were complement dependent and were prevented by complement inhibition. Values are shown as means \pm s.d. $(n=6) ;{ }^{*} P<0.01$ vs saline treated.

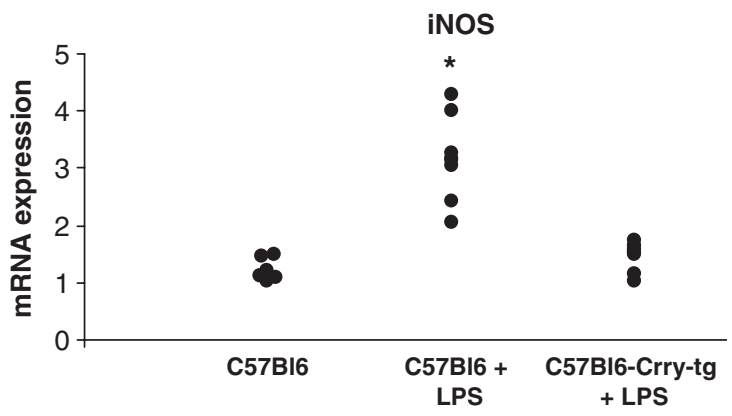

Figure 4 Complement inhibition reduces LPS-mediated upregulation of brain iNOS expression. Expression of iNOS was assessed by qRT-PCR and normalized to the expression of 18S. LPS caused a significant increase in iNOS expression, which was reduced by complement inhibition. Data from individual animals are shown with the means as horizontal lines; ${ }^{*} P<0.01$ vs control.

C57BL6 mice, which was reduced by complement inhibition $(1.2 \pm 0.2,3.16 \pm 0.8$ and $1.46 \pm 0.2$-fold in C57BL6, LPStreated C57BL6 and C57BL6-Crry-tg mice, respectively; $P=0.05$; Figure 4).

\section{Complement Inhibition Reduces Gliosis in Endotoxemia} In the control mice, glial expression in cortex was very low (Figure 5a). On LPS treatment, many activated lectin-positive microglia (upper panel) and GFAP-positive astroglia (lower panel) were observed in the cortex (Figure $5 b$ ). Inset shows microglial and astroglial cells at higher magnification $(\times 40$ under oil). Glial cells were mainly present in the cortex and the hippocampus. The number of activated glia was significantly reduced by complement inhibition (Figure $5 c$ ).

\section{Complement Inhibition Reduces Apoptosis in Lupus Brains}

There is evidence indicating that apoptosis occurs in the brains of LPS-treated mice. Complement proteins ${ }^{32}$ and 

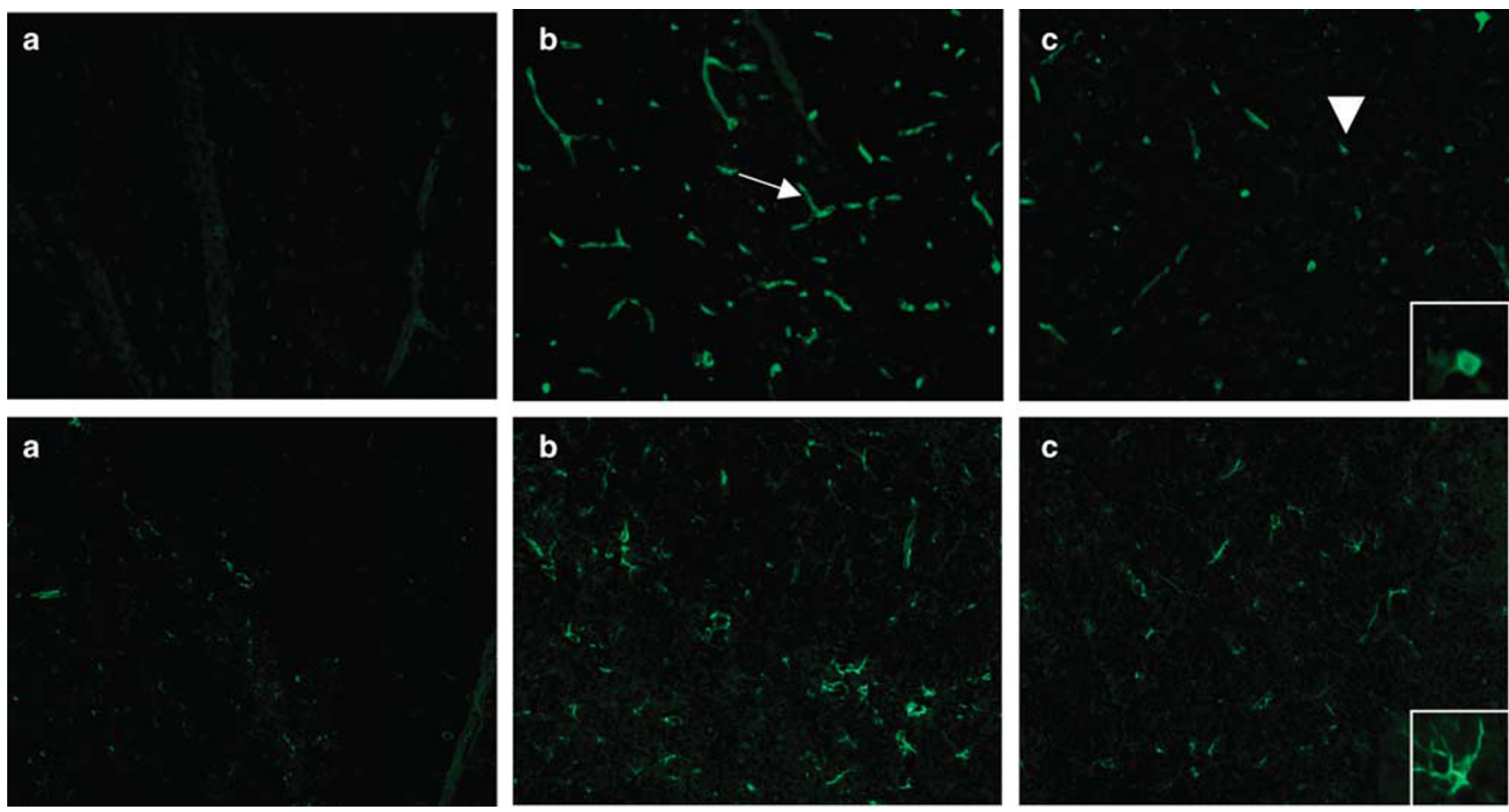

Figure 5 Glial activation is reduced by complement inhibition. Sections from C57BL6 mice, C57BL6 mice treated with LPS and C57BL6-Crry-tg mice treated with LPS were stained with lectin (upper panel) and GFAP (lower panel). Representative sections shown clearly indicate a significant increase in the number of activated glial cells on treatment with LPS (b) compared with controls (a). This activation was reduced by complement inhibition (c). The inset shows glial cells at $\times 40$ magnification, under oil.

activation products along with $\mathrm{NO}$ and other cytokines induce apoptosis in neurons, which are remarkably sensitive. ${ }^{33-36}$ The relevance of the complement system to apoptosis in the brains of mice was studied using the DNA fragmentation technique (Figure 6) and substantiated by the TUNEL staining technique. There were a significant number of apoptotic cells in the brain sections in LPS-treated mice (Figure 7b) compared with control C57BL6 mice (Figure 7a), which was significantly reduced in littermate Crry transgenic animals (Figure $7 \mathrm{c}$ ). This indicates that the complement cascade may be one of the important mechanisms contributing to the observed neurodegeneration. A double labeling technique showed that the anti-neurofilament-stained neurons (Figure 7e) contained TdT-labeled apoptotic nuclei (Figure 7d), confirming neuronal apoptosis in these mice. Concomitant with the decrease in apoptosis in Crry-tg mice, there was reduced TLR4 expression in these mice. Futhermore, p-Akt (phosphorylated at serine 473), which is known to be critical for the control of cell survival, was increased in Crry-tg mice compared with the C57BL6 mice treated with LPS (Figure 8).

\section{Endotoxemia Increases Brain AQP4 Expression and Water Content}

Cerebral edema can occur as a result of many factors including activation of the complement cascade $^{37}$ and increased expression of TNF- $\alpha .{ }^{38}$ To determine whether edema occurred in the brains of mice administered LPS, we mea-

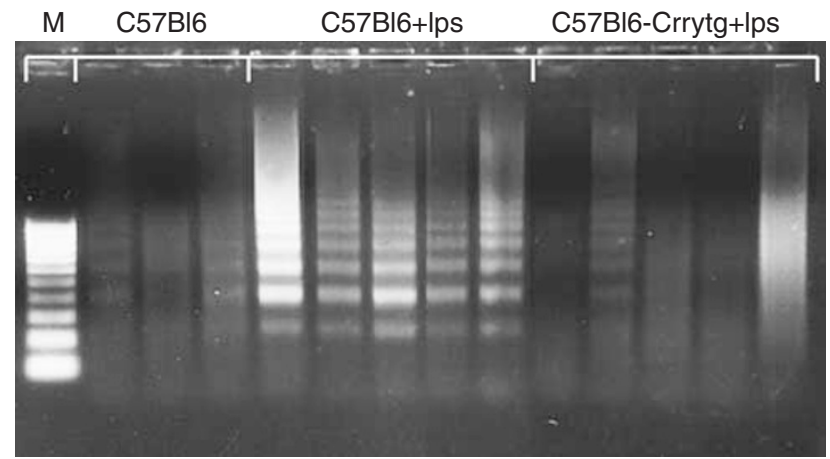

Figure 6 Crry reduces endotoxemia-induced apoptosis in the brain. DNA was isolated from brains and subjected to LM-PCR to assess apoptotic DNA laddering. Significant apoptosis was observed in the brains of the wild-type mice $8 \mathrm{~h}$ after LPS injection, which was considerably reduced by complement inhibition. Each lane contains brain tissue harvested from an individual animal $8 \mathrm{~h}$ after injection of LPS, with separate wild-type controls studied in each experiment.

sured water content in the brains of control and LPS-treated mice. Water content in the brain tissue was increased approximately $10 \%(75.3-82.5 \%)$ in wild-type mice treated with LPS compared with those controls treated with saline (Figure 9). Increased expression of Crry reduced the water content to $78 \%$. Since AQP4 is the main water channel protein in brain, we studied its expression in endotoxemic mice. Concomitant with the LPS-induced increase in brain 

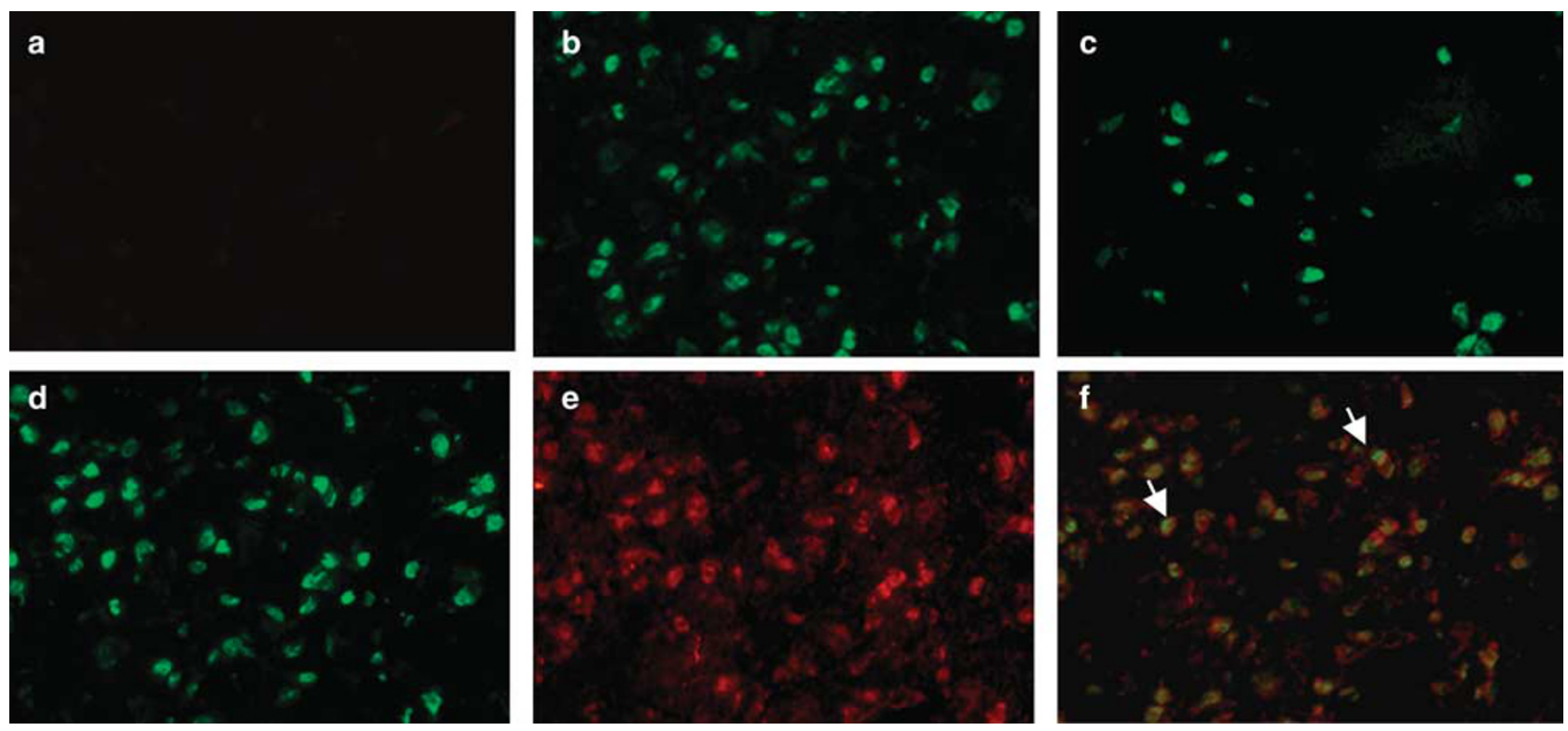

Figure 7 Apoptosis induced by LPS treatment primarily occurs in neuronal cells. TUNEL staining was performed to define the localization of the apoptotic cells in LPS-treated brain. A few apoptotic cells were present in the cortex of control mice (a), while a substantial number of apoptotic cells were observed in the cortex of LPS-treated mice (b). Apoptosis was definitely reduced by complement inhibition (c). Cellular localization of apoptotic cells was determined by a double labeling technique. Biotin-TdT in apoptotic nuclei was identified with streptavidin-FITC (d), and neurons were stained with rabbit anti-mouse neurofilament $\mathrm{Ab}$, followed by rhodamine-conjugated anti-rabbit $\lg \mathrm{G}(\mathbf{e})$. The merge of both pictures is shown in (f).

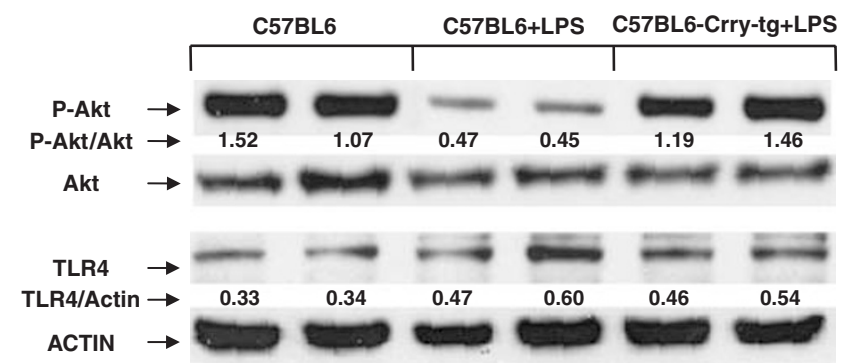

Figure 8 Complement activation alters the expression of survival regulators in the brains of endotoxemic mice. The expression of survival factors Akt and p-Akt were assessed by western blotting along with TLR4. They were normalized to the housekeeping protein, actin. The expression of TLR4 in the LPS brain was significantly reduced by complement inhibition. In contrast, p-Akt expression was increased in C57BL6-Crry-tg mice compared with LPS-treated C57BL6 mice.

water content, the mRNA expression of AQP4 was significantly increased in these mice compared with control mice treated with saline (Figure 9), and partially abrogated by complement inhibition. These results support that systemic LPS administration upregulates AQP4, which may be responsible for the observed edema, in a manner similar to that induced by intranigral injection of LPS in rats. ${ }^{18}$

\section{DISCUSSION}

Encephalopathy is a common feature in sepsis, occurring in $\sim 25 \%$ of patients often before failure of other organs such as the kidney, liver or lung. Patients with septic encephalopathy

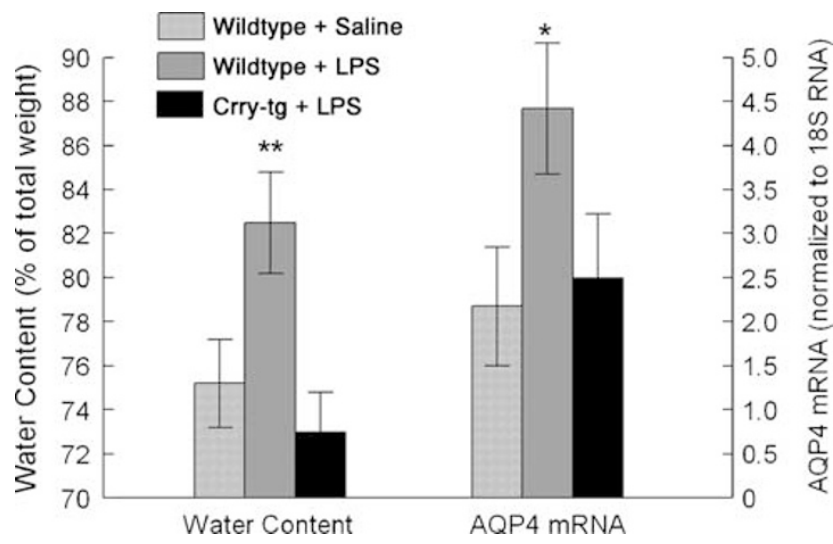

Figure 9 Complement plays an important role in the regulation of brain water content in endotoxemic mice. Brain water content was measured as described in Materials and Methods. Data are expressed as \% water content in brains. Complement inhibition by Crry prevented the increase of water content in the endotoxin brain. Concomitantly, mRNA expression of AQP4 was increased on LPS treatment compared with controls of the brain. This increase was definitely prevented by complement inhibition. mRNA was quantified by qRT-PCR ( $n=6$ per group) normalized to $18 \mathrm{~S}$. Data are shown as means \pm s.d.; ${ }^{*} P<0.05$ vs other two groups; ${ }^{*} P<0.01$.

have a higher mortality rate compared with those without brain involvement, likely reflecting the severity of the disease and the direct adverse effects of brain involvement. The pathogenesis of septic encephalopathy is multifactorial and includes circulatory and metabolic derangements, ${ }^{39}$ inflammation and infections ${ }^{40}$ of the brain. 
In this study, we evaluated the response of the brain to endotoxemia. Arguably, inflammation and apoptosis are the two of the most important underlying causes of septic encephalopathy. ${ }^{41}$ After systemic administration of LPS, a profound upregulation of mRNA for $\mathrm{C} 3$ and downregulation of mRNA for Crry was observed in the brain. Given the intrinsic ability of the brain to synthesize $\mathrm{C} 3,{ }^{3}$ increased C3 deposition in the brains of LPS-injected mice could be due to its increased synthesis or increased transport into the brain. C3 synthesis and deposition remained at normal levels in Crry-tg mice, suggesting that complement activation maybe one of the mechanisms that cause brain pathology in endotoxemia. Once complement activation occurs, a number of downstream effects will be initiated. It increases permeability of the $\mathrm{BBB},{ }^{42}$ induces expression of cell adhesion molecules in endothelial cells and astrocytes, thereby favoring leukocyte infiltration and inflammatory brain damage, ${ }^{43}$ and can play an important role in inducing apoptosis. ${ }^{44}$

LPS treatment induced glial activation based on increased cell number, increased expression of cell-specific-targeted antigen and morphological features of activation. Further, LPS treatment causes increased infiltration of neutrophils into the brain parenchyma. ${ }^{45}$ Activated neutrophils produce NO by iNOS. Further, in neurons, signaling of TNF- $\alpha$ through its receptor, TNFR1 downregulates survival signals ${ }^{46,47}$ and stimulates the expression of iNOS, which generates the cytotoxic oxygen radical NO. ${ }^{35}$ Both TNF- $\alpha$ and NO were shown to be involved in neuronal death and damage. ${ }^{48}$ The upregulation of iNOS expression after systemic LPS administration was reduced in Crry-tg mice, suggesting that complement activation-induced NO production may be one of the mechanisms that can lead to brain pathology in septic encephalopathy.

The environment of the brain is maintained by the BBB. Increased protein levels found in the cerebrospinal fluid of septic encephalopathy patients suggest that their BBB is compromised. ${ }^{49}$ Similarly, in rodents with systemic sepsis, the altered entry of neutral amino acids into the brain indicated that the integrity of the BBB was disrupted. ${ }^{50}$ Intrathecal injection of LPS into piglet brains induced both pathological and biochemical changes, including edema, increased $\mathrm{BBB}$ permeability and infiltration of neutrophils into the brain. In our study, a 10\% increase in brain water content was found in mice that were treated with LPS. Brain water content is regulated by AQP4 present in astrocytic end feet and around blood vessels. Following MCAO, $\mathrm{AQP} 4^{-1-}$ mice had less cerebral edema and a better clinical outcome than controls, suggesting that AQP4 expression enhances edema fluid formation. Further, edema has been shown to be complement dependent. ${ }^{51}$ Therefore, the increased brain water content observed in our study may be attributed to complement-dependent upregulated AQP4 expression.

Recently it was demonstrated that complement regulates TLR signaling in vivo. ${ }^{52}$ Our results suggest that in endotoxemic brains, complement activation leads to altered expression of TLR4 and subsequent alterations in TNF- $\alpha$, iNOS and AQP4. Although the resulting inflammation, apoptosis and edema largely resolve with time in surviving experimental animals, there is evidence for lasting cognitive and morphological abnormalities well after the septic episode. ${ }^{53}$ The results of our study suggest that targeting activation of the complement system may be a viable therapeutic avenue in septic encephalopathy.

\section{ACKNOWLEDGEMENT}

This work was supported by NIH Grants R01DK041873 and R01DK055357 to RJQ.

1. Papadopoulos MC, Davies DC, Moss RF, et al. Pathophysiology of septic encephalopathy: a review. Crit Care Med 2000;28:3019.

2. Green R, Scott LK, Minagar A, et al. Sepsis associated encephalopathy (SAE): a review. Front Biosci 2004;9:1637.

3. van BJ, Elward K, Gasque P. Activation of complement in the central nervous system: roles in neurodegeneration and neuroprotection. Ann N Y Acad Sci 2003;992:56.

4. Nataf $\mathrm{S}$, Stahel PF, Davoust $\mathrm{N}$, et al. Complement anaphylatoxin receptors on neurons: new tricks for old receptors? Trends Neurosci 1999;22:397.

5. Rancan M, Morganti-Kossmann MC, Barnum SR, et al. Central nervous system-targeted complement inhibition mediates neuroprotection after closed head injury in transgenic mice. J Cereb Blood Flow Metab 2003;23:1070.

6. de Boer JP, Creasey AA, Chang A, et al. Activation of the complement system in baboons challenged with live Escherichia coli: correlation with mortality and evidence for a biphasic activation pattern. Infect Immun 1993;61:4293.

7. Stahel PF, Barnum SR. Bacterial meningitis: complement gene expression in the central nervous system. Immunopharmacology 1997;38:65.

8. Smedegard G, Cui LX, Hugli TE. Endotoxin-induced shock in the rat. A role for C5a. Am J Pathol 1989;135:489.

9. Bengtson A, Heideman M. Anaphylatoxin formation in sepsis. Arch Surg 1988;123:645.

10. Heideman M, Norder-Hansson B, Bengtson A, et al. Terminal complement complexes and anaphylatoxins in septic and ischemic patients. Arch Surg 1988;123:188.

11. Nakae $\mathrm{H}$, Endo $\mathrm{S}$, Inada $\mathrm{K}$, et al. Serum complement levels and severity of sepsis. Res Commun Chem Pathol Pharmacol 1994;84:189.

12. Muller-Eberhard HJ. The membrane attack complex of complement. Annu Rev Immunol 1986;4:503.

13. Alexander JJ, Lim A, He C, et al. Renal, central nervous system and pancreatic overexpression of recombinant soluble Crry in transgenic mice. A novel means of protection from complement-mediated injury. Immunopharmacology 1999;42:245.

14. He C, Alexander JJ, Lim A, et al. Production of the rat complement regulator, Crry, as an active soluble protein in Pichia pastoris. Arch Biochem Biophys 1997;341:347.

15. Foley $S$, Li B, Dehoff $M$, et al. Mouse Crry/p65 is a regulator of the alternative pathway of complement activation. Eur J Immunol 1993;23:1381.

16. Badaut J, Lasbennes F, Magistretti PJ, et al. Aquaporins in brain: distribution, physiology, and pathophysiology. J Cereb Blood Flow Metab 2002;22:367.

17. Agre $P$, King LS, Yasui $M$, et al. Aquaporin water channels-from atomic structure to clinical medicine. J Physiol 2002;542:3.

18. Tomas-Camardiel M, Venero JL, De Pablos RM, et al. In vivo expression of aquaporin-4 by reactive microglia. J Neurochem 2004;91:891.

19. Bohatschek M, Werner A, Raivich G. Systemic LPS injection leads to granulocyte influx into normal and injured brain: effects of ICAM-1 deficiency. Exp Neurol 2001;172:137.

20. Dereski MO, Chopp M, Knight RA, et al. The heterogeneous temporal evolution of focal ischemic neuronal damage in the rat. Acta Neuropathol (Berl) 1993;85:327. 
21. Bolton SJ, Anthony DC, Perry VH. Loss of the tight junction proteins occludin and zonula occludens-1 from cerebral vascular endothelium during neutrophil-induced blood-brain barrier breakdown in vivo. Neuroscience 1998;86:1245.

22. Garcia JH, Liu KF, Yoshida Y, et al. Influx of leukocytes and platelets in an evolving brain infarct (Wistar rat). Am J Pathol 1994;144:188.

23. Wispelwey B, Hansen EJ, Scheld WM. Haemophilus influenzae outer membrane vesicle-induced blood-brain barrier permeability during experimental meningitis. Infect Immun 1989;57:2559.

24. Khan RZ, Badr KF. Endotoxin and renal function: perspectives to the understanding of septic acute renal failure and toxic shock. Nephrol Dial Transplant 1999;14:814.

25. Gardenfors A, Nilsson F, Skagerberg G, et al. Cerebral physiological and biochemical changes during vasogenic brain oedema induced by intrathecal injection of bacterial lipopolysaccharides in piglets. Acta Neurochir (Wien) 2002;144:601.

26. Radzivil GG, Beloborodov VB, Bronikin I. [Hemodynamics and rheologic properties of the blood in meningococcemia associated with meningitis and complicated by septic shock and intracranial hypertension]. Anesteziol Reanimatol 1990;28:33.

27. Stahel PF, Frei K, Eugster HP, et al. TNF-alpha-mediated expression of the receptor for anaphylatoxin C5a on neurons in experimental Listeria meningoencephalitis. J Immunol 1997;159:861.

28. Quigg RJ, He C, Lim A, et al. Transgenic mice overexpressing the complement inhibitor crry as a soluble protein are protected from antibody-induced glomerular injury. J Exp Med 1998;188:1321.

29. Kubes P, Kanwar S, Niu XF, et al. Nitric oxide synthesis inhibition induces leukocyte adhesion via superoxide and mast cells. FASEB J 1993;7:1293.

30. Semmler A, Okulla T, Sastre M, et al. Systemic inflammation induces apoptosis with variable vulnerability of different brain regions. J Chem Neuroanat 2005;30:144.

31. Hersch M, Scott JA, Izbicki G, et al. Differential inducible nitric oxide synthase activity in circulating neutrophils vs mononuclears of septic shock patients. Intensive Care Med 2005;31:1132.

32. Uwai M, Terui $Y$, Mishima $Y$, et al. A new apoptotic pathway for the complement factor B-derived fragment Bb. J Cell Physiol 2000;185:280.

33. Chao CC, Hu S, Molitor TW, et al. Activated microglia mediate neuronal cell injury via a nitric oxide mechanism. J Immunol 1992;149:2736.

34. Leist M, Volbracht C, Kuhnle S, et al. Caspase-mediated apoptosis in neuronal excitotoxicity triggered by nitric oxide. Mol Med 1997;3:750.

35. Heneka MT, Loschmann PA, Gleichmann M, et al. Induction of nitric oxide synthase and nitric oxide-mediated apoptosis in neuronal PC12 cells after stimulation with tumor necrosis factor-alpha/ lipopolysaccharide. J Neurochem 1998;71:88.

36. Peterson PK, Hu S, Anderson WR, et al. Nitric oxide production and neurotoxicity mediated by activated microglia from human versus mouse brain. J Infect Dis 1994;170:457.

37. Alexander JJ, Bao L, Jacob A, et al. Administration of the soluble complement inhibitor, Crry-lg, reduces inflammation and aquaporin 4 expression in lupus cerebritis. Biochim Biophys Acta 2003;1639:169.
38. Sanni LA, Jarra W, Li C, et al. Cerebral edema and cerebral hemorrhages in interleukin-10-deficient mice infected with Plasmodium chabaudi. Infect Immun 2004;72:3054.

39. Rus HG, Kim LM, Niculescu Fl, et al. Induction of $\mathrm{C} 3$ expression in astrocytes is regulated by cytokines and Newcastle disease virus. J Immunol 1992;148:928.

40. Busquets $\mathrm{S}$, Alvarez $\mathrm{B}, \mathrm{Van} \mathrm{RM}$, et al. Increased uncoupling protein-2 gene expression in brain of lipopolysaccharide-injected mice: role of tumour necrosis factor-alpha? Biochim Biophys Acta 2001;1499:249.

41. Zudaire E, Martinez A, Cuttitta F. Adrenomedullin and cancer. Regul Pept 2003;112:175.

42. Tsao N, Hsu HP, Wu CM, et al. Tumour necrosis factor-alpha causes an increase in blood-brain barrier permeability during sepsis. J Med Microbiol 2001;50:812.

43. Alexander JJ, Jacob A, Vezina $\mathrm{P}$, et al. Absence of functional alternative complement pathway alleviates lupus cerebritis. Eur J Immunol 2007;37:1691-1701.

44. Akassoglou K, Bauer J, Kassiotis G, et al. Oligodendrocyte apoptosis and primary demyelination induced by local TNF/p55TNF receptor signaling in the central nervous system of transgenic mice: models for multiple sclerosis with primary oligodendrogliopathy. Am J Pathol 1998;153:801.

45. Connolly Jr ES, Winfree CJ, Springer TA, et al. Cerebral protection in homozygous null ICAM-1 mice after middle cerebral artery occlusion. Role of neutrophil adhesion in the pathogenesis of stroke. J Clin Invest 1996;97:209.

46. Venters HD, Dantzer R, Kelley KW. Tumor necrosis factor-alpha induces neuronal death by silencing survival signals generated by the type I insulin-like growth factor receptor. Ann N Y Acad Sci 2000;917:210.

47. Venters HD, Dantzer R, Kelley KW. A new concept in neurodegeneration: TNFalpha is a silencer of survival signals. Trends Neurosci 2000;23:175.

48. Hidaka K, Mitsuyama T, Furuno $\mathrm{T}$, et al. The role of nitric oxide in human pulmonary artery endothelial cell injury mediated by neutrophils. Int Arch Allergy Immunol 1997;114:336.

49. Voigt $\mathrm{K}$, Kontush A, Stuerenburg HJ, et al. Decreased plasma and cerebrospinal fluid ascorbate levels in patients with septic encephalopathy. Free Radic Res 2002;36:735.

50. Jeppsson B, Freund HR, Gimmon Z, et al. Blood-brain barrier derangement in sepsis: cause of septic encephalopathy? Am J Surg 1981;141:136.

51. Xi G, Hua Y, Keep RF, et al. Brain edema after intracerebral hemorrhage: the effects of systemic complement depletion. Acta Neurochir Suppl 2002;81:253.

52. Zhang X, Kimura Y, Fang C, et al. Regulation of Toll-like receptormediated inflammatory response by complement in vivo. Blood 2007;110:228-236.

53. Semmler A, Frisch C, Debeir T, et al. Long-term cognitive impairment, neuronal loss and reduced cortical cholinergic innervation after recovery from sepsis in a rodent model. Exp Neurol 2007;204:733. 